\title{
Investigation Into Environmentally Friendly Alternative Cleaning Processes for Hybrid Microcircuits to Replace Vapor Degreasing With 1,1,1 Trichloroethane
}

Federal Manufacturing \& Technologies

B. E. Adams

RECEIVED

KCP-613-5850

MAY 121997

Published February 1997

Final Report

Approved for public release; distribution is unlimited.
OSTI

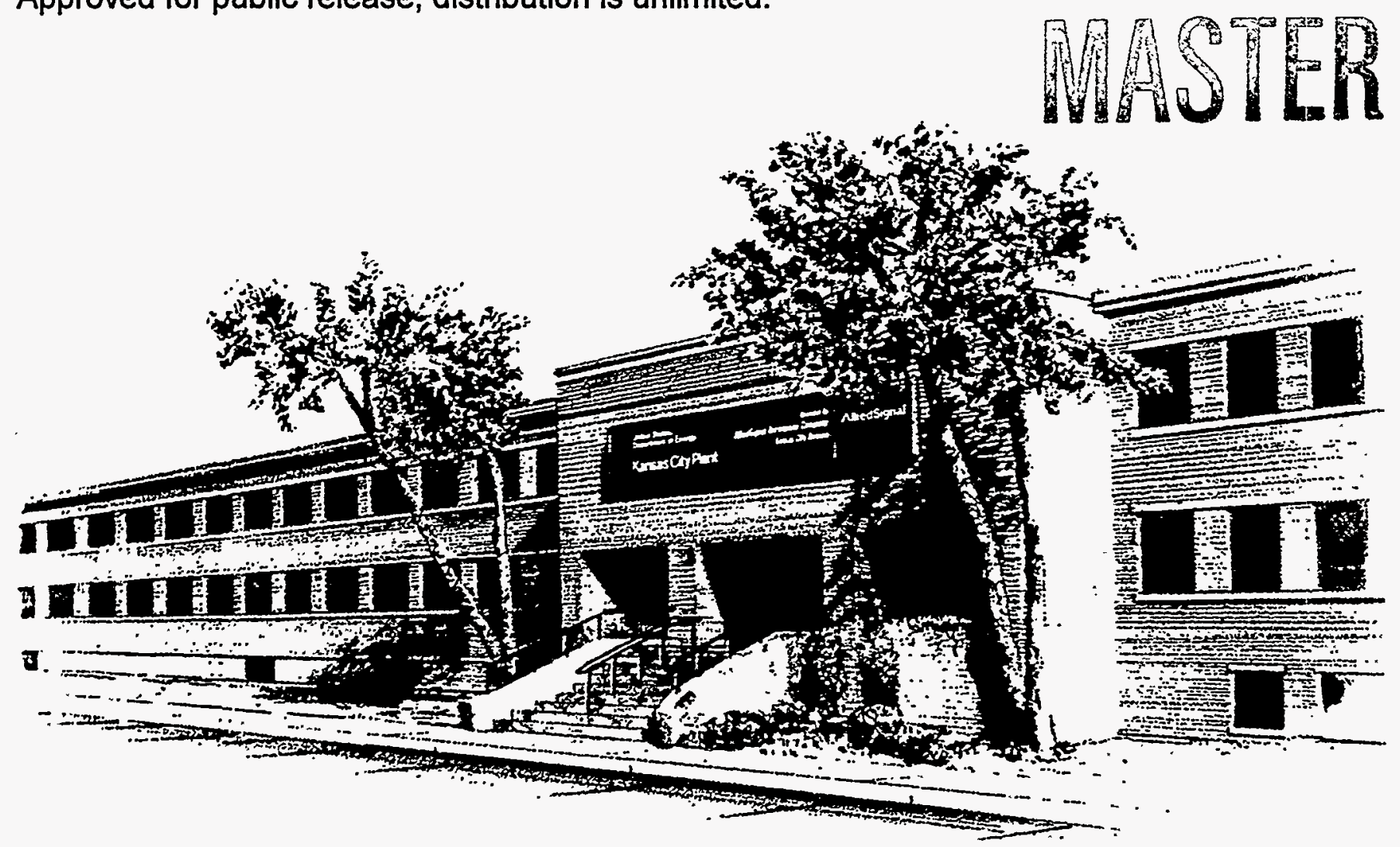

DISTRIBUTION OF THIS DOCUMENT IS UNLIMITED 


\section{DISCLAIMER}

This report was prepared as an account of work sponsored by an agency of the United States Government. Neither the United States Government nor any agency thereof, nor any of their employees, makes any warranty, express or implied, or assumes any legal liability or responsibility for the accuracy, completeness, or usefulness of any information, apparatus, product, or process disclosed, or represents that its use would not infringe privately owned rights. Reference herein to any specific commercial product, process, or service by trade names, trademark, manufacturer, or otherwise, does not necessarily constitute or imply its endorsement, recommendation, or favoring by the United States Government or any agency thereof. The views and opinions of authors expressed herein do not necessarily state or reflect those of the United States Government or any agency thereof.

All data prepared, analyzed and presented has been developed in a specific context of work and was prepared for internal evaluation and use pursuant to that work authorized under the referenced contract. Reference herein to any specific commercial product, process or service by trade name, trademark, manufacturer, or otherwise, does not necessarily constitute or imply its endorsement, recommendation, or favoring by the United States Government, any agency thereof or AlliedSignal Inc.

Printed in the United States of America.

This report has been reproduced from the best available copy.

Available to DOE and DOE contractors from the Office of Scientific and Technical Information, P. O. Box 62, Oak Ridge, Tennessee 37831; prices available from (615) 576-8401, FTS 626-8401.

Available to the public from the National Technical Information Service, U. S. Department of Commerce, 5285 Port Royal Rd., Springfield, Virginia 22161. 


\section{DISCLAIMER}

This report was prepared as an account of work sponsored by an agency of the United States Government. Neither the United States Government nor any agency thereof, nor any of their employees, make any warranty, express or implied, or assumes any legal liability or responsibility for the accuracy, completeness, or usefulness of any information, apparatus, product, or process disclosed, or represents that its use would not infringe privately owned rights. Reference herein to any specific commercial product, process, or service by trade name, trademark, manufacturer, or otherwise does not necessarily constitute or imply its endorsement, recommendation, or favoring by the United States Government or any agency thereof. The views and opinions of authors expressed herein do not necessarily state or reflect those of the United States Government or any agency thereof. 


\section{DISCLAIMER}

Portions of this document may be illegible in electronic image products. Images are produced from the best available original document. 
Approved for public release; distribution is unlimited.

\section{INVESTIGATION INTO ENVIRONMENTALLY FRIENDLY ALTERNATIVE CLEANING PROCESSES FOR HYBRID MICROCIRCUITS TO REPLACE VAPOR DEGREASING WITH 1,1,1 TRICHLOROETHANE}

B. E. Adams

Published February 1997

Final Report

B. E. Adams, Project Leader

Project Team:

G. W. Bohnert

T. E. Hand

B. W. Lenhardt 



\section{Contents}

Section

Page

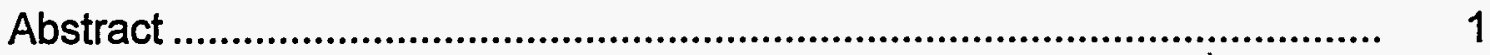

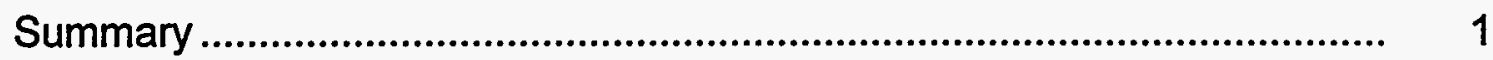

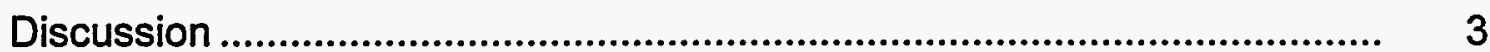

Scope and Purpose ............................................................................ 3

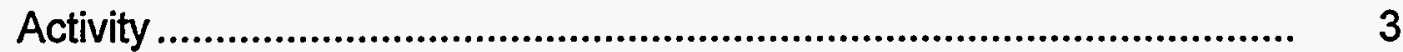

Introduction ...........................................................................

Background on Cleaning Chemistry ........................................... 6

Experimentation ....................................................................... 7

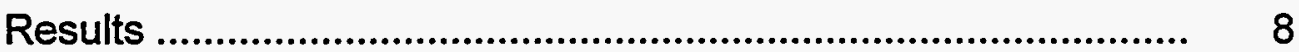

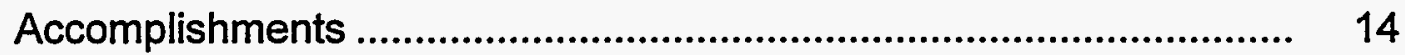

Recommendations ......................................................................... 15

Appendices

A. Summary of Results Obtained by Material Engineering for the Cleaning Ability of Various Solvents on Solder Flux Residue...... 16

B. Memorandums Discussing the Method of Incorporating the d-Limonene Cleaning Process 


\section{Illustrations}

Figure

Page

1 Spacing Between Substrate and Surface Mount Devices ................... 5

2 Sapphire Hybrid Spray-Cleaned at 80 psig for 20 Minutes Using d-Limonene

3 Sapphire Hybrid Cleaned by Microdrop Aqueous Saponification Process

4 Backside of LCCs Removed From Networks After Aqueous Saponification Cleaning.

\section{Tables}

Number

Page

1 Average Meseran Residual Rosin and Omega Meter Results for Various Flux Removal Solvents on Drag-Soldered PWBs Using Kester 1544 Solder Flux.

2 Visual Results for Various Cleaning and Assembly Methods

9

3 Meseran Measurements Made Under Surface Mount Components for Hybrids Cleaned With Three Different Solvents.

4 Residual Rosin and Omega meter Testing Results for Hybrids

5 Compatibility Results for Various Components and Materials Exposed to Solutions for Five Days.

A-1 Omega meter 600 SMD Test Results for Removal of Kester 197 Flux.

A-2 Meseran and Visual Results of the Removal of Kester 197 Flux From Copper Panels

A-3 Measure of Residual Kester 197 Flux on Copper Panels Using Auger Spectroscopy 


\section{Abstract}

Two cleaning processes, one aqueous and one nonaqueous, were investigated as potential replacements for the vapor degreasing process using $1,1,1$ trichloroethane (TCA) for hybrid microcircuit assemblies. The aqueous process was based upon saponification chemistry. A $10 \%$ solution of either Kester 5768 or Armakleen 2001 , heated to $140^{\circ} \mathrm{F}$, was sprayed on the hybrid at $450 \mathrm{psig}$ and a flow rate of $5 \mathrm{gpm}$ through a specially designed nozzle which created microdroplets. The nonaqueous process was based upon dissolution chemistry and used d-limonene as the solvent in an immersion and spray process. The d-limonene solvent was followed by an isopropyl alcohol spray rinse to remove the excess $d$-limonene. The aqueous microdroplet process was found to be successful only for solder reflow profiles that did not exceed $210^{\circ} \mathrm{C}$. Furthermore, removal of component marking was a problem and the spray pressure had to be reduced to $130 \mathrm{psig}$ to eliminate damage to capacitor end caps. The d-limonene cleaning was found to be successful for solder reflow temperature up to $250^{\circ} \mathrm{C}$ when using a four-step cleaning process. The four steps included refluxing the hybrid at $80^{\circ} \mathrm{C}$, followed by soaking the hybrid in $d$-limonene which is heated to $80^{\circ} \mathrm{C}$, followed by spray cleaning at 80 psig with room temperature d-limonene, followed by spray cleaning at 80 psig with room temperature IPA was developed to remove residual flux from the hybrid microcircuits. This process was the most robust and most closely matched the cleaning ability of TCA.

\section{Summary}

Trichloroethylene (TCE) and 1,1,1 trichloroethane (TCA) have long been used as flux removal solvents for hybrid microcircuit assembly. These solvents were excellent cleaners, but the potential health risk associated with TCE and the o-zone-depleting nature of TCA have forced industry to look for less toxic, more environmentally friendly replacements. At AlliedSignal Federal Manufacturing \& Technologies (FM\&T), the materials engineering department ran many studies looking at the flux removal capability of several aqueous and nonaqueous solvents. All studies were run on coupons and looked at the solvency of Kester 135 flux in each of the solutions. From these data several candidates emerged as potential replacements for TCA in flux removal processes, with d-limonene appearing to be the best substitute.

For this project two cleaning processes, one aqueous and one nonaqueous, were investigated for the removal of residual flux from surface mount hybrid microcircuits. The aqueous process tested Kester 5768 and Armakleen 2001 in a heated high pressure spray cleaning application, The nonaqueous process tested d-limonene in three different cleaning processes: spray cleaning, centrifugal cleaning, and immersion cleaning (heated and unheated). All d-limonene cleaning processes were followed by an IPA spray rinse to remove residual d-limonene.

Two assembly methods were used during the evaluation. The first was soldering to reflowed solder bumps using flux as a tacking agent to hold the components in place. This left gross amounts of flux beneath each component, increasing the difficulty of flux removal. The second was placing components directly into solder paste and adding no additional flux. This minimized the amount of flux on the board and trapped beneath the components. However, 
placing components in paste led to the problem of solder balls trapped under components with low standoff heights. The solder ball issue limited the number of components that could be placed in paste. Both thick film (TKN) and thin film (TFN) surface mount hybrids were assembled. On TKNs, 63/37 Sn/Pb solder was used and on TFNs, 50/50 Pb/ln solder was used. The $\mathrm{Pb} / \mathrm{ln}$ solder presented an additional challenge because the peak reflow temperature reached $250^{\circ} \mathrm{C}$, which increased the difficulty of removing residual flux because of rosin polymerization.

Cleaning of surface mount hybrids was proven to be dependent upon the cleaning process, the solder reflow temperature, the part geometry, the component density, the component standoff height, and the operator's assembly techniques. No singular d-limonene process (meaning spray, immersion, or centrifugal only) could successfully remove residual solder flux from surface mount hybrids. Only when hybrids were refluxed at $80^{\circ} \mathrm{C}$, followed by soaking in heated d-limonene, followed by spraying with d-limonene, and finally spraying with isopropyl alcohol was flux successfully removed from the entire hybrid (including under LCCs). The aqueous high pressure spray cleaning process using a saponifier successfully removed the solder flux from under the LCCs when 63-37 $\mathrm{Sn}-\mathrm{Pb}$ solder was used. However, problems arose with the removal of ink marking on components and damage to end caps of the capacitors. Reducing the spray pressure to 130 psig resolved the capacitor end cap problems, but ink marking was still being removed. Aqueous saponification with DI water rinse could not remove even minimal amounts of residual flux when the reflow temperature for $50 / 50 \mathrm{~Pb} / \mathrm{ln}$ solder was used. That same residue could be removed by the four-step d-limonene/IPA process mentioned above. Placing components directly into solder paste to minimize flux usage could not be used for every component because of solder ball formation during reflow. The solder balls would get trapped under the low profile components and could short adjacent pins.

Based upon the results of the cleaning experiments, it is recommended that the assembly department incorporate the four-step d-limonene cleaning process for hybrid manufacturing. The four steps include

1) heated reflux of hybrids at $80^{\circ} \mathrm{C}$,

2) ten-minute soak in d-limonene heated to $80^{\circ} \mathrm{C}$,

3) spray clean with room temperature d-limonene for one minute at $80 \mathrm{psig}$, and

4) spray clean with room temperature IPA for one minute at $80 \mathrm{psig.}$

This process is the most robust and least operator dependent. Aqueous saponification cleaning could be incorporated on hybrids or PWAs provided the reflow temperature remains below $210^{\circ} \mathrm{C}$, component marking can be protected, and spray pressures are maintained at 130 psig. Refluxing hybrids heated to $80^{\circ} \mathrm{C}$ prior to cleaning will also improve the aqueous process. 


\section{Discussion}

\section{Scope and Purpose}

The purpose of this investigation was to develop environmentally conscious cleaning processes for the removal of flux residue from surface mount hybrid microcircuits. The new processes were to meet or exceed the current environmental regulations and maintain the reliability and manufacturability of the product to current levels.

\section{Activity}

\section{Introduction}

Trichloroethylene (TCE) and 1,1,1 trichloroethane (TCA) have long been used as flux removal solvents for hybrid microcircuit assembly. These solvents were excellent cleaners, but the potential health risk associated with TCE and the o-zone-depleting nature of TCA have forced industry to look for less toxic, more environmentally friendly replacements. Switching to an environmentally friendly cleaning process is no different than any other process modifieation. The key to a smooth conversion is through an analysis of existing cleaning process, questioning the key areas of concerns related to cleaning, and demonstrating that the alternative process meets the requirements of the concerns.

In selecting a cleaning process the key questions which must be answered are the following:

1. Is cleaning necessary?

2. What contaminants are being removed (organic, inorganic salts, particulate)?

3. Can the range of contaminants be reduced?

4. Can the amount be reduced?

5. What materials are being cleaned?

6. What are the part geometry and assembly method?

7. Is there a cleaning standard? (How clean is clean?)

8. Are the materials compatible with the cleaning solution?

9. How does cleaning affect other production processes?

10. What is the environmental impact of new cleaning process?

11. What is the health and safety impact for the operator?

12. What is the economic impact of the new process?

In this investigation the first seven questions were used to determine what cleaning processes to investigate, and the remaining five questions would be answered after the investigation was completed.

\section{Is cleaning necessary?}

Although it has been argued that leaving rosin flux behind poses no threat to the hybrid microcircuit, the fear is that trapped ionic contaminants may be present in the residual flux; and upon exposure to humid air, water will attach to the circuit and a conductive path will form on the surface of the network. Therefore, it is important to remove all contamination from the board surface, so that no conductive paths can form. 


\section{What contaminants are being removed?}

For surface mount hybrid assembly, the purpose in cleaning is to remove residual rosin flux left after soldering. The typical residues which exist after soldering include

- unmodified, oxidized, and polymerized rosin,

- mono- and dicarboxylic acids,

- metallic halides and abietates,

- metallic mono- and dicarboxylates,

- hydrochloric acid (RA and RMA fluxes only),

- quaternary ammonium halides (for RA and RMA fluxes only).

\section{Can the range or amount be reduced?}

The range of contaminants has already been minimized to improve cleaning. Currently, only RMA and pure rosin fluxes are being used in soldering processes in the assembly department (Radar Hybrid Microcircuit). Furthermore, all soldering processes have been optimized for the desired solder reflow profiles for the given components and substrates. The quantity of flux being used is an issue. Steps have been taken to minimize the use of solder flux, such as adding a nitrogen cover gas to the solder belt and using premixed solder paste and flux. This has helped to reduce the quantity used.

\section{What materials are being cleaned?}

The materials being cleaned include gold, gold-plated kovar, aluminum oxide (ceramic), fired thick film gold paste, ceramic capacitors, thick film resistors, tinned $\mathrm{Cu} / \mathrm{P}$ edge clips, $\mathrm{Sn} / \mathrm{Pb}$ solder, $\mathrm{Pb} / \mathrm{ln}$ solder, green cover glass, tantalum nitride, and titanium palladium thin film resistors.

\section{What are the part geometry and assembly method?}

For any rosin flux removal techniques, the quality of cleaning can vary enormously with part geometry and assembly method for a given flux chemistry. The part geometry can be broken down to include component density, component size, component standoff height, and solvent flow restrictions under surface mount components due to solder joints. For hybrids built in two assembly departments (Radar Hybrid Microcircuit and Surface Mount HMC Assembly), the standoff height ranges from 0.001 inch (1 mil) to 0.003 inch (3 mil). Figure 1 shows the 3-mil clearance that a 20-pin LCC has when soldered to a ceramic network. Two assembly methods were used during evaluation. The first was soldering to reflowed solder bumps using flux as a tacking agent to hold components on the solder bumps. This left gross amounts of flux beneath each component, increasing the difficulty of flux removal. The second was placing components directly into solder paste and adding no additional flux. This minimized the amount of flux on the board but created a solder ball problem for low profile surface mount components. Both thick film (TKN) and thin film (TFN) surface mount hybrids were assembled. On TKNs, $63 / 37 \mathrm{Sn} / \mathrm{Pb}$ solder was used and on TFNs, 50/50 Pb/ln solder was used. The Pb/ln solder presents a second challenge in that the peak reflow temperature reaches $250^{\circ} \mathrm{C}$. The higher peak temperature increases the difficulty in removing residual solder flux because of the polymerization of the rosin. 


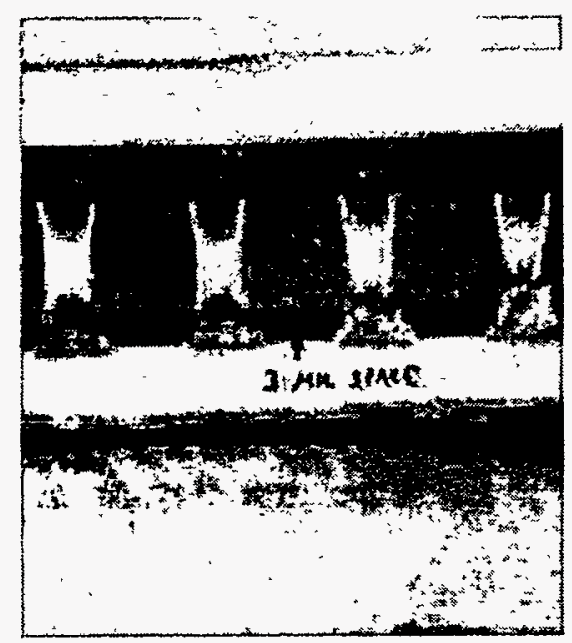

Figure 1. Spacing Between Substrate and Surface Mount Devices

\section{Is there a cleaning standard?}

A cleaning standard has been developed in industry for omega meter testing and is described in the following paragraph. AlliedSignal developed its own cleaning standard known as meseran testing. Its description follows the omega meter discussion. Several testing methods were used in this investigation including meseran testing, omega meter testing, residual rosin testing, Auger analysis, and visual inspection at 30X under normal and ultraviolet (UV) lighting. Results of the tests were compared to cleaning results using TCA to determine the ability of the processes and solvents. A brief description of each test method follows.

Omega meter testing - This is an indirect test method which measures the amount of ionic contamination removed from a test panel (or printed wiring assembly [PWA]), using a heated solution of $75 \%$ IPA and $25 \%$ DI water. The test panel is submerged in the solution for 10 minutes during which time the solution's resistivity is measured. The change in the resistivity is converted to $\mu \mathrm{g} \mathrm{NaCl} / \mathrm{in}^{2}$ equivalents. The board or test panel is considered clean if the total equivalence of sodium chloride is less than $10 \mu \mathrm{g} \mathrm{NaCl} / \mathrm{in}^{2}$. The concern of this test is that not all of the ionic contamination on the test panel is removed from the board.

Meseran testing - This test procedure uses an evaporative rate analysis technique to detect organic contamination. A carbon-14 tagged radioactive chemical is deposited on the test board surface, and a Geiger counter measures the rate of evaporation for a given time period. A clean surface yields a low meseran number, indicating a quick evaporation of the tagged solvent. As a general rule based on experience, a count of $\mathbf{2 0 0}$ or higher indicates a contaminated surface and counts less than 100 indicate a clean surface. The concern with this test is that surface roughness affects the evaporation rate of the solvent which can lead to wide variation of results among tested PWAs. 
Auger electron and $X$-ray photoelectron spectroscopy - This is a direct test method that measures the surface elemental composition of the top 50 angstroms of the test panel. This is not necessarily a quantitative test but instead qualitative. Auger electron spectroscopy is accomplished by exciting the sample with a 3 to $20 \mathrm{keV}$ electron beam and measuring the kinetic energy of the Auger electrons which are emitted from the sample surface. The technique for chemical analysis of surfaces can be visualized as a two-step process. First, a high energy photon, generated by an electron beam, ejects an eleciron from a core orbital (1s) of an atom. Second, an electron falls from a higher energy orbital to fill the vacancy, thereby releasing sufficient energy to eject a second photoelectron from one of the higher energy orbitals. The ejected photoelectron has a measurable characteristic energy which relates to the element from which was ejected.

Residual rosin testing - This is an indirect test method which measures the amount of abieatic acid remaining on the test coupon. The test board is soaked in a heated solution of $98.9 \%$ IPA, $1 \%$ phosphoric acid, and $0.1 \%$ water solution for 1 hour. A sample of the solution is then placed in a UV analyzer which is set at $242 \mathrm{~nm}$. Absorbtion reading at this wavelength coincides with the amount of abeiatic acid in solution, which gives an indication of the residual rosin on the board surface in $\mu \mathrm{g} / \mathrm{in}^{2}$. Again, the concern is that not all contamination is_removed from the board during analysis.

\section{Background on Cleaning Chemistry}

Two types of cleaning processes were evaluated in this study. Dissolution chemistry using d-limonene and isopropyl alcohol was tested as well as saponification chemistry in an aqueous solution. A brief description of each follows.

\section{Dissolution Chemistry}

Intermolecular forces are of primary importance in explaining the solubility of a substance. Dissolution of a solid in a liquid is like melting a solid. The orderly crystal structure of the solid is destroyed, and the result is the formation of a more disorderly arrangement of the molecules in solution. In the process of dissolving, the molecules or ions must be separated from each other, and energy must be supplied for both changes. The energy required to overcome lattice energies and intermolecular or interionic attractions comes from the formation of new attractive forces between the solute and the solvent. The addition of heat to the solution will improve solubility of the solute. The heat adds the required energy to break the intermolecular bonds and dissolve the solute. The simple rule for dissolution chemistry is "like dissolves like." Rosin fluxes are typically $97 \%$ abieatic and pimaric acids of the diterpene carboxylic type.

D-limonene, being in the terpene family, is chemically similar to flux, and therefore is a good solvent for flux. However, d-limonene may not remove all the thermal breakdown components of flux after soldering. Following the d-limonene with IPA will improve cleaning by dissolving a wider range of contaminants, including ionic residues left by activators used in RMA and RA fluxes. For dissolution chemistry (d-limonene), the process parameters which must be optimized are time, mild heating, and mechanical agitation. 


\section{Saponification Chemistry}

Aqueous saponification cleaning technology has been in use since the 1960 s. Simply put, saponification is the chemical conversion of rosin flux to a water-soluble soap using an alkaline amine. When rosin flux is combined with monoethanolamine, it forms a soluble soap as shown in the chemical equation below.

$\underset{\substack{\mathrm{C}_{19} \mathrm{H}_{29} \mathrm{COOH} \\ \text { Rosin }}}{\mathrm{H}_{2} \mathrm{NCH}_{2} \mathrm{CH}_{2} \mathrm{OH}} \underset{\text { monoethanolamine }}{\mathrm{H}_{2}} \rightarrow \underset{\text { water-soluble soap }}{\mathrm{C}_{19} \mathrm{H}_{29} \mathrm{COOH}_{2} \mathrm{CH}_{2} \mathrm{NH}_{2}}+\mathrm{H}_{2} \mathrm{O}$

The reaction requires the addition of heat to push it to completion. The solution must be heated to between $140^{\circ} \mathrm{F}$ and $160^{\circ} \mathrm{F}$ for optimum results. Once the rosin acids are converted to watersoluble soaps, a DI water rinse will remove the contamination.

Quaternary ammonium halides are activators used in flux to improve the solderability. The two most commonly used are dimethyl- and diethylammonium chloride. Theoretically, at soldering temperature, they decompose to $\mathrm{HCl}$ gas and dimethyl or diethyl amine gas. In practice, the decomposition will be only partial and residues of the unmodified activator and the two reaction gases will be found trapped in the rosin. These are all water soluble, and the alkalinity associated with saponifiers will neutralize any $\mathrm{HCl}$, rendering it harmless. The metallic chlorides and organometallic salts that can form during soldering (often referred to as white residue) are insoluble in chlorinated solvents, have limited solubility in water, and are typically removable with water using complexing agents. Process parameters for saponification include time, mechanical agitation, temperature, and solution concentration.

\section{Experimentation}

Materials Engineering ran many studies looking at the flux removal capability of several aqueous and nonaqueous solvents. All studies were run on copper and aluminum panels and looked at the solvency ability of each solution when used in a spray cleaning or ultrasonic immersion cleaning process. The cleaning ability of each solvent was assessed by meseran testing, visual inspection under long wave UV light, and some omega meter testing. From these data several candidates emerged as potential replacements for TCA in flux removal processes, with d-limonene appearing to be the best substitute. A sample of the data collected by Materials Engineering is included in Appendix A.

The first effort of this investigation was to verify Materials Engineering's results on drag soldered printed wiring board assemblies and IR belt soldered ceramic substrates. Omega meter testing, meseran testing, and residual rosin testing were run for each cleaning solution. Auger surface analysis was run on networks cleaned in TCA and a 5\% solution of Kester 5768 in DI water. Concurrently, based on the Materials Engineering's results of the original study mentioned above, d-limonene was being tested on scrap production surface mount parts using three different cleaning processes, spray cleaning, centrifugal cleaning, and immersion cleaning, all followed by IPA spray rinse. Aqueous saponification in a high pressure spray process was also tested on scrap production surface mount hybrids. 
Initially, the primary method for determining the cleaning ability of each process used on the scrap production parts was visual inspection of parts under $30 \mathrm{X}$ magnification. Any evidence of residual flux indicated insufficient cleaning by the process. To ease the verification of cleaning beneath components, hybrids were assembled on sapphire substrates. Using sapphire allowed the visual examination under components without destructively removing the component.

Ultimately omega meter testing and residual rosin testing were done on TKN and TFN hybrids which were belt soldered using 63/37 $\mathrm{Sn} / \mathrm{Pb}$ solder on TKNs and 50/50 Pb/ln solder on TFNs. In this study the efficacy of TCA, d-limonene followed by IPA, and aqueous saponification with DI water rinse was tested and compared. Meseran testing was also run on the TKN hybrids assembled by solder bump method and cleaned by d-limonene/IPA spray cleaning, TCA vapor degreasing, and microdrop aqueous saponification cleaning. The meseran measurements were taken from underneath surface mounted LCCs which had been mechanically removed.

Finally, a short-term compatibility study was run for each material or component that would be exposed to the cleaning solution. The study included a five-day soak of the material in the solution. Changes in mass, coloration, and volume were measured.

\section{Results}

\section{Phase I}

The results of the initial screening tests run on printed wiring boards can be seen in Table 1.

Table 1. Average Meseran Residual Rosin and Omega Meter Results for Various Flux Removal Solvents on Drag-Soldered PWBs Using Kester 1544 Solder Flux

\begin{tabular}{|l|c|c|c|}
\hline \multicolumn{1}{|c|}{ Solvent } & Meseran No. & $\begin{array}{c}\text { Residual Rosin } \\
\left(\mu \mathrm{g} / \mathrm{in}^{2}\right)\end{array}$ & $\begin{array}{c}\text { Ionic Residue } \\
\left(\mu \mathrm{g} / \mathbf{i n}^{2}\right)\end{array}$ \\
\hline Alpha 2110 & 812 & 1232 & 9.1 \\
\hline Aquanox 101 & 873 & 921 & 9.4 \\
\hline Aquanox SSA & 848 & 1157 & 5.5 \\
\hline Axarel 38 & 327 & 587 & $\mathrm{nt}$ \\
\hline Bioact EC Ultra & 638 & 849 & $\mathrm{nt}$ \\
\hline Detergent 8 & 1020 & 973 & $\mathrm{nt}$ \\
\hline Ethyl Lactate & 1121 & 1064 & $\mathrm{nt}$ \\
\hline Exxate 600 & 604 & 112 & $\mathrm{nt}$ \\
\hline Flux Off II & 305 & 221 & $\mathrm{nt}$ \\
\hline lonox FC & 792 & 1348 & 4.2 \\
\hline Kester Biokleen 5768 & 824 & 1058 & $\mathrm{nt}$ \\
\hline D-limonene & 377 & 68 & 13.1 \\
\hline Loncoterge 255 & 923 & 1255 & $\mathrm{nt}$ \\
\hline Prozone SL9 & 1031 & 509 & $\mathrm{nt}$ \\
\hline Re-Entry KNI 2000 & 645 & 144 & $\mathrm{nt}$ \\
\hline THFA & 995 & 914 & 22.3 \\
\hline TCE & 67 & 26 & 11.0 \\
\hline Vi Chem 600A & 930 & 315 & \\
\hline
\end{tabular}


The initial screening indicated that d-limonene was the closest match to TCE, followed by Flux Off II, Exxate 600, and Re-Entry KNI 2000. From these results, d-limonene was tested on scrap production hybrids. Three processes were investigated, aspirated spray cleaning at 80 psig, centrifugal cleaning, and room temperature immersion cleaning. Hybrids built on sapphire substrates were visually examined for flux residue after cleaning. The hybrid assembly technique used was to apply solder flux to reflowed solder bumps on the network to hold the LCC in place during soldering. This technique traps large amounts of flux under the LCCs. A photograph of a d-limonene spray cleaned hybrid assembled on a sapphire network showing gross contamination remaining can be seen in Figure 2. The visual results for each cleaning processes and assembly method are listed in Table 2.

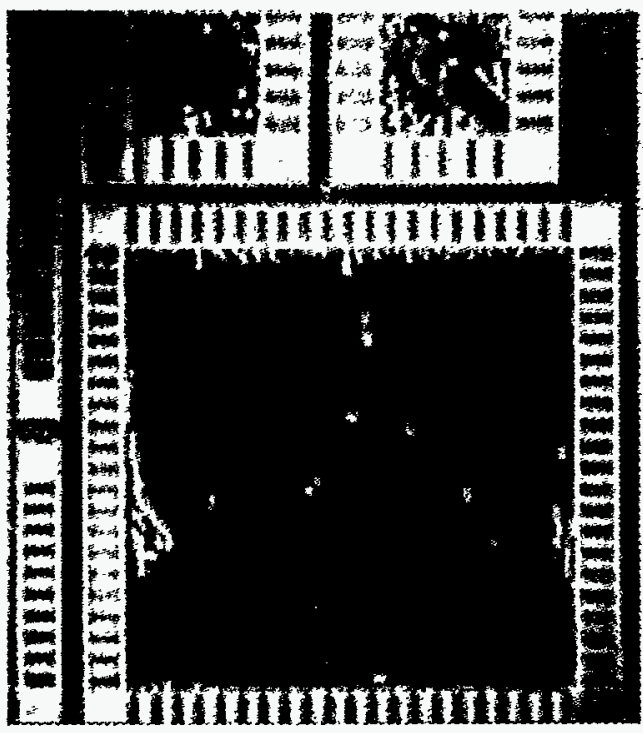

Figure 2. Sapphire Hybrid Spray-Cleaned at 80 psig for 20 Minutes Using d-Limonene

Table 2. Visual Results for Various Cleaning and Assembly Methods

\begin{tabular}{|l|l|c|l|}
\hline Cleaning Method & Assembly Method & Network Type & \multicolumn{1}{|c|}{ Visual Results } \\
\hline d-limonene spray & Attach to solder bumps & TKN & $\begin{array}{l}\text { Flux remained } \\
\text { under components }\end{array}$ \\
\hline d-limonene spray & Attach in solder paste & TKN & All flux removed \\
\hline d-limonene spray & Attach in solder paste & TFN & All flux removed \\
\hline Aqueous saponification & Attach to solder bumps & TKN & All flux removed \\
\hline Aqueous saponification & Attach in solder paste & TKN & All flux removed \\
\hline Aqueous saponification & Attach in solder paste & TFN & $\begin{array}{l}\text { Residual flux rosin } \\
\text { remains }\end{array}$ \\
\hline d-limonene immersion & Attach to solder bumps & TKN & $\begin{array}{l}\text { Flux remained } \\
\text { under components }\end{array}$ \\
\hline d-limonene centrifugal & Attach to solder bumps & TKN & $\begin{array}{l}\text { Flux remained } \\
\text { under components }\end{array}$ \\
\hline
\end{tabular}


10

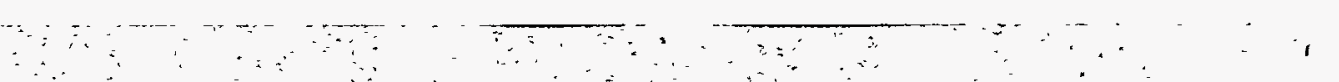


Results of meseran testing used to compare d-limonene spray cleaning to vapor degreasing in trichloroethane and spray cleaning with an aqueous saponifier (Kester 5768) are given in Table 3. The area under the surface mounted LCC was examined during the testing.

Table 3. Meseran Measurement Made Under Surface Mount Components for Hybrids Cleaned With Three Different Solvents

\begin{tabular}{|l|c|c|}
\hline Solvent & Meseran No. (LCC 1) & Meseran No. (LCC 2) \\
\hline TCA & 225 & 325 \\
\hline D-limonene & 700 & 725 \\
\hline Kester 5768 & 400 & 50 \\
\hline
\end{tabular}

The preliminary results indicated that although d-limonene appeared to be the best solvent, it would not sufficiently clean under the components of the surface mount hybrids by spray cleaning, centrifugal cleaning, or room temperature immersion cleaning.

The next step was to investigate the aqueous saponification process using Biokleen 5768. A special spray nozzle was designed that forms microdroplets when operated at $450 \mathrm{psig}$ and a flow rate of $5 \mathrm{gpm}$. D-limonene could not be used in this process because of its combustibility. When tested on hybrids built on sapphire, the high pressure, aqueous saponification process removed all the residual solder flux from beneath the LCCs as seen in Figure 3. When tested on production hybrids, the LCCs were mechanically removed from the substrates after cleaning and visually examined. Figure 4 shows the results of spray cleaning for various amounts of time. The visual examination revealed that high pressure spray cleaning with an aqueous saponifier successfully cleaned surface mount hybrids which had been soldered with 63/37 $\mathrm{Sn} / \mathrm{Pb}$ solder and Kester 197 flux after spraying for 10 minutes minimum.

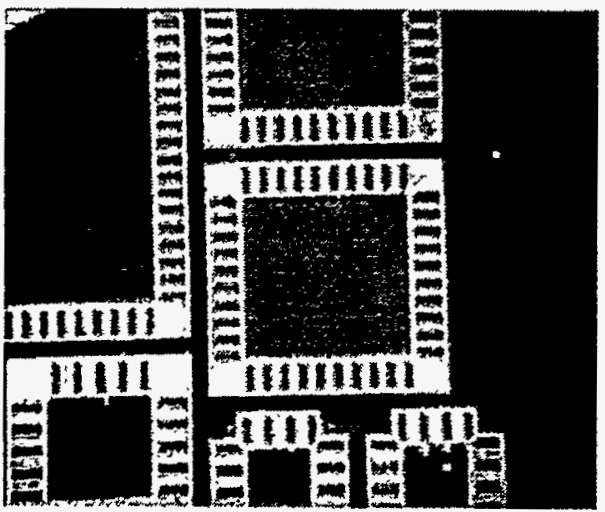

Figure 3. Sapphire Hybrid Cleaned by Microdrop Aqueous Saponification Process

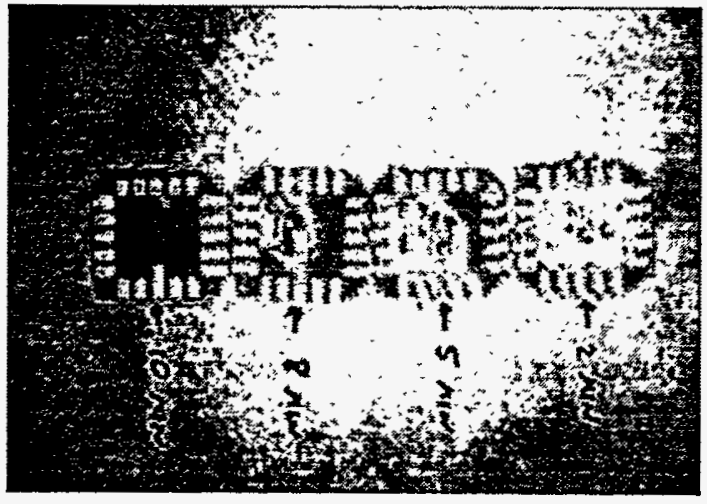

Figure 4. Backside of LCCs Removed From Networks After Aqueous Saponification Cleaning 


\section{Phase II}

With the success of the aqueous saponification on the sapphire substrates and scrap production parts, the process was implemented in the assembly department on development build. Running the microdrop process on a daily basis revealed the shortcomings of the process which are listed below:

1. Damaged end caps of the ceramic capacitors due to the high pressure, high flowrate spray.

2. Removal of many of the component's marking.

3. Inability to remove flux residue from thin film networks solder with $50-50 \mathrm{~Pb} / \mathrm{ln}$ solder.

None of these problems could be eliminated with further characterization studies of the microdrop process. For these reasons, the process was eliminated, and d-limonene cleaning was reinvestigated for the common radar. The implementation of the d-limonene cleaning was coordinated with the design lab. Appendix B contains a brief history of the discussions and studies run during the implementation process.

The original problem with d-limonene cleaning was the inability to remove residual fluxfrom under the surface mount components. The original technique used to attach the components was to use flux as a tacking agent for the components on previously reflowed solder bumps. This technique trapped large quantities of flux under the components. To reduce the amount of flux used during soldering, components with standoff heights greater than five mils were placed in solder paste, and no additional flux was added. This helped to reduce the quantity of flux residue and aided the cleaning process. However, LCCs and surface mount capacitors were still attached to reflowed solder bumps and were held in place with flux. These components could not be placed in paste because solder balls would get trapped under components during solder reflow. Some of these solder balls were large enough to short adjacent pins. Also, rework processes still required copious amounts of flux which increased the difficulty of cleaning. To overcome the difficult cleaning, a four-step cleaning process was developed. The process included

1. dripping flux on a contaminated hybrid heated to $80^{\circ} \mathrm{C}$,

2. soaking in $\mathrm{d}$-limonene heated to $80^{\circ} \mathrm{C}$ for 10 minutes,

3. spraying with room temperature d-limonene for one minute at $80 \mathrm{psig}$, and

4. spraying with room temperature isopropyl alcohol for one minute at 80 psig.

The most important steps in the cleaning process were the heated refluxing and heated immersion soak. As new flux is dripped onto a heated hybrid (which has just been soldered), convection currents form within the added flux because of the temperature gradient through the liquid. These currents have a scrubbing effect, and with the additional heat energy they loosen and dislodge the flux residue, bringing it into solution with the new added flux. The tight spacing under the surface mount devices pulls the added flux under the components by capillary action. The added flux continues to form eddy currents under the components because of the temperature gradient and remove the baked-on flux under the devices. Following the refluxing with a heated d-limonene soak, the additional flux which now contains the baked-on flux residue is easily removed. The heated soak also pulls flux out from under the components because of the flux's concentration gradient. The last two spray cleaning steps of the process are to ensure that all flux and d-limonene are removed from the hybrid surface. 
The cleaning ability of the new d-limonene process was compared to the cleaning ability of trichloroethane and Biokleen 5768 (aqueous saponifier) on actual production hybrids. The results of these tests can be seen in Table 4.

Table 4. Residual Rosin and Omega Meter Testing Results for Hybrids

\begin{tabular}{|c|c|c|c|c|}
\hline HMC & $\begin{array}{l}\text { Cleaning } \\
\text { Method }\end{array}$ & $\begin{array}{l}\text { Residual } \\
\text { Rosin }\left(\mu \mathrm{g} / \mathrm{in}^{2}\right)\end{array}$ & $\begin{array}{l}\text { Omega Meter } \\
\left(\mu \mathrm{g} / \mathrm{in}^{2}\right) \text { FM\&T }\end{array}$ & $\begin{array}{l}\text { Omega Meter } \\
\left(\mu \mathrm{g} / \mathrm{in}^{2}\right) \text { SNL/NM }\end{array}$ \\
\hline TKNC & $\begin{array}{l}\text { TCA } \\
\text { TCA } \\
\text { TCA } \\
\text { d-limonene } \\
\text { d-limonene } \\
\text { d-limonene } \\
\text { aqueous }\end{array}$ & $\begin{array}{l}4 \\
14 \\
16 \\
13 \\
20 \\
96 \\
0\end{array}$ & $\begin{array}{l}1 \\
0 \\
0.9 \\
7.8 \\
3.8 \\
1.4 \\
5.5\end{array}$ & $\begin{array}{l}2.67 \\
5.81 \\
0.9\end{array}$ \\
\hline$\overline{T F N V}$ & $\begin{array}{l}\text { TCA } \\
\text { TCA } \\
\text { TCA } \\
\text { d-limonene } \\
\text { d-limonene } \\
\text { d-limonene } \\
\text { aqueous }\end{array}$ & $\begin{array}{l}8 \\
11 \\
6 \\
65 \\
145 \\
51 \\
666\end{array}$ & $\begin{array}{l}\text { Omega meter } \\
\text { failure at FM\&T } \\
\text { required testing } \\
\text { to be done at } \\
\text { SNL/NM }\end{array}$ & $\begin{array}{l}1.32 \\
1.32 \\
1.06\end{array}$ \\
\hline TFNF & d-limonene & 62 & & 1.75 \\
\hline TFN T & d-limonene & 82 & & 3.5 \\
\hline TFN M & d-limonene & 215 & & 1.04 \\
\hline
\end{tabular}

The data indicated that d-limonene closely matched the cleaning ability of TCA.

\section{Compatibility Testing}

Short-term compatibility testing of the hybrid microcircuits was run in d-limonene and Biokleen 5768. The short-term testing consisted of soaking each component and network in the solvent for up to five days. Table 5 shows the results of the testing.

To test the long-term compatibility of a radar to residual d-limonene, two complete radar channels were assembled and run through a one-year aging and temperature cycle study. The channels were electrically tested periodically during the aging, and at the conclusion of the study the radars were d-tested. No degradation of the electrical characteristics or mechanical integrity of the radar channel was found. 
Table 5. Compatibility Results for Various Components and Materials Exposed to Solutions for Five Days

\begin{tabular}{|l|l|l|}
\hline Material & d-limonene & Biokleen 5768 \\
\hline Aluminum & no reaction & etching of aluminum \\
\hline Ceramic & no reaction & no reaction \\
\hline Gold-plated kovar & no reaction & no reaction \\
\hline TKN & no reaction & no reaction \\
\hline TFN & no reaction & no reaction \\
\hline $\mathrm{Sn} / \mathrm{Pb}$ solder & no reaction & etching of $\mathrm{Pb}$ \\
\hline $\mathrm{Pb} / \mathrm{ln}$ solder & no reaction & etching of $\mathrm{Pb}$ \\
\hline $\mathrm{Cu} / \mathrm{P}$ edge clips & no reaction & etching of $\mathrm{Cu}$ \\
\hline $\mathrm{LCC}$ & no reaction & no reaction \\
\hline
\end{tabular}

\section{Accomplishments}

Cleaning of surface mount hybrids has proven to be dependent upon the cleaning process, the solder reflow temperature, the component density, the standoff height of components from the substrate surface, and the operator's assembly techniques. The quantity of flux remaining can affect the ability of the cleaning process by restricting flow under surface mount components. This is also true for densely populated substrates and for surface mount devices with low standoff height. A high reflow temperature such as occurs when reflowing $\mathrm{Pb}-\mathrm{In}$ solder causes flux to polymerize and become unreactive. Before a solvent can remove solder flux residue, it must be delivered by a process that gets the solvent to the necessary areas and contains the energy required to break chemical bonds and take the residue into solution.

In the first study it was found that $d$-limonene cleaning by spray, immersion, and centrifugal cleaning all done at room temperature was unsuccessful at removing solder flux from under LCCs when excessive amounts of flux were used during soldering. Gross contamination remained on each part after cleaning for up to 20 minutes. On the other hand, aqueous saponification cleaning using the microdrop technology successfully removed the solder flux for the same situation. However, aqueous saponification damaged capacitor end caps, removed component marking, and could not remove even a minimal amount of residual flux when the reflow temperature for $\mathrm{Pb} / \mathrm{ln}$ solder was used. Because of the damage caused by the microdrop process and the inability of aqueous saponification to remove flux residue after $\mathrm{Pb} / \mathrm{ln}$ soldering, the process and the solvent were eliminated as a potential replacement for vapor degreasing in TCA.

D-limonene was shown to be a good solvent for rosin flux; however, the geometry of surface mount hybrids made flux removal very difficult with the available processes. Although the microdrop process increased the available energy for cleaning, it also caused mechanical damage to the hybrid. Also, d-limonene is a combustible material and can not be used in the microdrop machine. To overcome d-limonene's inability to remove residual flux at room temperature, a heated refluxing step and heated d-limonene soak were added to the cleaning process. The added flux and heat increased the available energy for dissolution of the flux residue. Heating d-limonene required the installation of an explosion-proof hood. Furthermore, an upper limit of $80^{\circ} \mathrm{C}$ was set for the $d$-limonene. The addition of heat greatly improved the cleaning process using d-limonene. 
The short-term compatibility studies run with d-limonene showed it to be compatible with all materials. The saponifier solution reacted with the $\mathrm{Sn} / \mathrm{Pb}$ and $\mathrm{Pb} / \mathrm{ln}$ solder, the $\mathrm{Cu} / \mathrm{P}$ edge clips, and any aluminum handling fixtures. The etching is a surface reaction and does not impair the mechanical or electrical properties of the materials. Rinsing the hybrids for as little as 15 seconds will stop the reaction of saponifier with $\mathrm{Cu}, \mathrm{Pb}$, or Al. All other materials were compatible with the aqueous solution.

\section{Recommendations}

Based on the results of the experimentation, it is recommended that the assembly department use the four-step heated refluxing, heated immersion cleaning in d-limonene followed by spray rinsing with d-limonene, and last spray cleaning with isopropyl alcohol to remove excess solvent from the hybrids. This recommendation is based on the current soldering practices within the department and because both thin and thick film hybrids are assembled in the area. The aqueous saponification cleaning process using the microdrop technology is too aggressive and damages components during operation. Furthermore, residual flux could only be removed from thick film hybrids and printed wiring boards using $63 / 37 \mathrm{Sn} / \mathrm{Pb}$ solder when using the aqueous saponifier. 


\section{Appendix A}

Summary of Results

\section{Obtained by Material Engineering \\ for the Cleaning Ability of Various Solvents \\ on Solder Flux Residue}


Table A-1. Omega Meter 600 SMD Test Results for Removal of Kester 197 Flux

\begin{tabular}{|l|c|}
\hline Solvent & Contamination Level $\left(\mu \mathrm{g} \mathrm{NaCl} / \mathrm{in}^{2}\right)^{\star}$ \\
\hline Act 100 & $0.73 \pm 0.35$ \\
\hline Kester 5769 (5\% solution in DI water) & 0 \\
\hline Exxate 1000 & $0.07 \pm 0.06$ \\
\hline Bioact EC-7 & 0 \\
\hline Bioact EC-7/lsopropyl Alcohol & 0 \\
\hline Isopropyl Alcohol & $1.47 \pm 0.12$ \\
\hline Trichloroethylene/lsopropyl Alcohol & $0.13 \pm 0.06$ \\
\hline
\end{tabular}

*9913000 Specification Acceptance Limit $=10 \mu \mathrm{g} \mathrm{NaCl} / \mathrm{in}^{2}$

Table A-2. Meseran and Visual Results of the Removal of Kester 197 Flux From Copper Panels

\begin{tabular}{|l|l|l|l|}
\hline Solvent & Spray Time (s) & Meseran No. & Visual Exam (UV) \\
\hline TCE & 60 & 0 & $\begin{array}{l}\text { Removed after 15 s } \\
\text { spray }\end{array}$ \\
\hline Exxate 1000 & 60 & 7 & $\begin{array}{l}\text { Removed after 45 s } \\
\text { spray }\end{array}$ \\
\hline Bioact EC-7. & 30 & 0 & $\begin{array}{l}\text { Removed after 45 s } \\
\text { spray }\end{array}$ \\
\hline Kester 5769 & 480 & 12 & $\begin{array}{l}\text { Slight remained after } \\
15 \text { minute spray }\end{array}$ \\
\hline Act-100 & 120 & 0 & $\begin{array}{l}\text { Slight remained after } \\
15 \text { minute spray }\end{array}$ \\
\hline IPA & 600 & 197 & $\begin{array}{l}\text { Moderate remained } \\
\text { after 15 minute spray }\end{array}$ \\
\hline
\end{tabular}

Table A-3. Measure of Residual Kester 197 Flux on Copper Panels Using Auger Spectroscopy (Atomic weight percent of contaminant on surface)

\begin{tabular}{|l|c|c|c|c|}
\hline Solvent & Spray Time (s) & $\begin{array}{l}\text { Contaminant } \\
\text { on Bare Cu }\end{array}$ & $\begin{array}{l}\text { Contaminant on } \\
\text { Cu/solder } \\
\text { Interface }\end{array}$ & $\begin{array}{l}\text { Contaminant } \\
\text { on Solder }\end{array}$ \\
\hline TCE/IPA & 30 & 4.03 & 1.8 & 0.25 \\
\hline Exxate 1000 & 30 & 5.78 & 2.17 & 0.26 \\
\hline Bioact EC-7 & 30 & 3.06 & 2.82 & 0.33 \\
\hline Kester 5769 & 300 & 3.43 & 2.49 & 0.35 \\
\hline Act 100 & 90 & 2.76 & 1.19 & 0.34 \\
\hline IPA & 300 & 6.07 & 3.77 & 0.13 \\
\hline
\end{tabular}


Appendix B

\section{Memorandums Discussing the Method of Incorporating the d-Limonene Cleaning Process}


Date: January 25, 1994

To: Distribution

From:

Subject: Minutes of

HMC and PWA Cleanirig meeting, January 25,1994.

A meeting was held at $K C D$ to discuss changing the cleaning process on HMC's from Aqueous cleaning to d-Limonene cleaning.

The purpose of the meeting was to determine if changing over to d-Limonene cleaning on . HMC's was feasible and if so what riteded to be done to insure its success.

The meeting began with short presentations . explaining the present Aqueous cleaning process and a chronology of how the cleaning process was developed. It was stated that Aqueous cleaning of Thick Film HMC's proved to be much easier than Thin Film HMC's. This is due to the fact that the 50/50 lead/indium solder used on TFN's is reflowed at a higher temperature than the 63/37 tin/lead solder used on TKN's.

During the beginning of HMC build it was determined that aqueous cleaning was not adequate for flux removal. A quick evaluation of d-limonene spray cleaning on a sample of eight networks (four TFN and four TKN) gave very good visual indications that it cleaned better than aqueous saponification.

Since the bigggest question was about the compatibility of TKN's and d-Limonene, was invited to discuss this issue. has been envolved in evaluating $d$-Limonene and the fireset. stated that they had indeed evaluated TKN's cleaned in d-Limonene. They had also ran extensive testing and concluded that there was no problem. This testing had included a significant number of TKN's (>150). 
Discussions during the day were very productive and yielded the following group concensous:

1. There are no apparent show stoppers or risks blocking the use of d-limonene on HMC's and PWA's.

2. It is $O K$ to change over to $d$-Limonene cleaning on HMC's and PWA's if there are no component compatability problems and the following is completed prior to change over.

a. The d-Limonene process is to be further characterized to eliminate the d-Liminone/flux soaking. It is desirable to only soak in d-Limonene.

b. The two vacuum ovens to be used for vacuum baking are to be characterized and found to be capable of at least maintaining PWA's and HMC's at $70 \mathrm{deg}$. C. for 15 minutes minimum.

c. The sequencing of temperature cycling and vacuum bake shall be changed to perform vacuum bake prior to temperature cycling.

3. Concurrent with build using d-Limonene cleaning sample HMC's will be build per the following matrix.

\begin{tabular}{|c|c|c|c|c||}
\hline \multicolumn{5}{|c|}{ Table 1: TESTING MATRIX } \\
\hline HMC & $\begin{array}{c}\text { TCA } \\
\text { CLEAN }\end{array}$ & $\begin{array}{c}\text { AQUEOUS } \\
\text { CLEAN }\end{array}$ & D-LIMONENE & TESTING \\
\hline C & 7 & 3 & 7 & $\begin{array}{l}3 \text { ea. Omega meter } \\
3 \text { ea. Residual Rosin } \\
1 \text { Charlie Cook }\end{array}$ \\
\hline V & 7 & 3 & 7 & $\begin{array}{l}3 \text { ea. Omega meter } \\
3 \text { ea. Residual Rosin } \\
1 \text { Charlie Cook }\end{array}$ \\
\hline F & 0 & 0 & 2 & $\begin{array}{l}1 \text { ea. Omega meter } \\
1 \text { ea. Residual Rosin }\end{array}$ \\
\hline T & 0 & 0 & 2 & $\begin{array}{l}1 \text { ea. Omega meter } \\
1 \text { ea. Residual Rosin }\end{array}$ \\
\hline
\end{tabular}


4. In addition, two V HMC's and two F-HMC's will be chosen from build. After normal electrical testing they will be aged a 160 deg. F. for 385 hours (unbiased) and retested. Two nonfunctional HMC's will also be subjected to this aging process and then tested for mechaniclal/chemical degradation. 
Sandia National Laboratories.

P. O. Box 5800

Albuquerque, New Mexico 87185-0368
Managed and Operated by Sandia Corporation a subsidiary of Martin Marietta Corporation

September 8, 1994

Allied Signal Inc./Kansas City Division

P. O. Box 419159

Kansas City, Missouri 64141-6159

On January 25, 1994, a meeting was held at AS-KCD to address a cleaning issue - At that time, an aqueous cleaner (Biokleen 5768) was successfully being used to clean RMA flux from thick film Hybrid Microcircuits (HMC's). When higher temperatures were required to build thin film HMC's, the aqueous cleaner was unable to remove the baked-on solder flux. As a result, d-limonene was recommended as a potential replacement for Biokleen 5768 for cleaning thin film HMC's. A cleaning matrix for qualifying d-limonene was established and included testing for ionic contamination.

Per your request, we have tested three thick film HMC's and seven thin film HMC's for ionic contamination. An Ionograph 500M was used to test the HMC's. The Ionograph measures the amount of ionic contamination extracted during a 15 minute test in a 75\% isopropyl alcohol/25\% de ionized water solution. The test gives an indication of how clean the HMC's are after processing. As a reference, Sandia soldering specification allows a maximum amount of ionic contamination per board of $10 \mu \mathrm{g} \mathrm{NaCl} / \mathrm{in}^{2}$.

Prior to testing in the Ionograph, all HMC's were visually inspected. Several components on each of the three thick film HMC's were sheared off for purposes of inspecting the underside of the component and the surface of the board. All three thick film HMC's inspected were visually clean. The thin film HMC's were also visually inspected prior to testing with the Ionograph using $12 \mathrm{X}$ magnification. None of the components on the thin film HMC's were sheared off as they were for the thick film HMC's. The following visual observations were made on the thin film HMC's:

\section{Visual Observations}

HMC-F 199000 - solder spots on the gold conductor were noted in different places, i.e. it appeared that the solder was meant to fill the via holes. There were also solder spots on the conductor where there were no vias present. No physical defects were observed on the circuit or components. Minor scratches were observed on the gold conductor on both sides of the HMC.

HMC-T 409225 \& 409285 - Some brown flux residue and brown spots were observed on HMC 409225 where the solder filled the via thru holes. Some brown flux residues were observed on HMC 409285 (not as much as 409225). Minor scratches were observed on the gold conductor on both sides of the HMC. 
HMC-M 409184 - Brown flux residue was observed on the top and on the edges of the ceramic substrate and on the solder filled vias. Minor scratches were observed on the gold conductor on both sides of the HMC.

HMC-V 408409 - Brown flux residues were observed on the solder filled vias. Brown flux residue was observed at the solder interface between the ceramic substrate and the gold plated Kovar substrate. A large solder blob was observed on top of the IC. Small solder spots were observed on the bottom side where they probably shouldn't be. Minor scratches were observed on the gold conductor on both sides of the HMC. Several small solder balls observed within the solder joints of all capacitors.

HMC-V 408415 - Gross flux residue was observed at the solder interface between the ceramic substrate and the gold plated Kovar substrate; also flux residues were observed on the areas where the via holes were filled with solder. White "crusty" residue was observed on all component solder joints and many small solder balls were also observed within the component solder joints. Small amounts of scratches on the gold conductor were observed on both sides of the HMC.

HMC-V 408416 - Minute amounts of flux residue were observed. Several small solder balls were observed within the solder joints on the IC and the capacitors. Very little scratching on the conductor was observed on the topside. A large blob of what looks like solder paste was observed on the top of the IC.

\section{Ionograph Results \& Discussion}

All thin and thick film HMC's tested were well below the maximum allowable ionic contamination limit of $10 \mu \mathrm{g} \mathrm{NaCl} /$ in $^{2}$. Some of the HMC's had visible flux residues, yet ionics were barely detected by the Ionograph 500M. HMC-V 408415 in particular had gross amounts of flux residue visible to the naked eye, yet only measured $1.06 \mu \mathrm{g}$ $\mathrm{NaCl} /$ in $^{2}$ contamination. Normally, circuit boards are tested for ionics directly after being cleaned. In this case, the HMC's were not tested for months after they had been assembled. This allowed time for the baked on flux; particularly on the thin film HMC's to dry to completion thus rendering the flux insoluble in the $75 \%$ IPA $25 \%$ DIW solution. HMC-V 408415 was retested in the Ionograph for an additional 30 minutes to see if some of the flux could be dissolved over a longer period of time. Negligible results were obtained.

The following ionic contamination results were obtained with the Ionograph 500M:

Ionograph 500M Data

Thick Film HMC's/C

\begin{tabular}{llll} 
Cleaned With & $\begin{array}{l}\text { Surface Area } \\
\text { in } 2\end{array}$ & $\begin{array}{l}\text { Total Equiv. } \\
\text { ugNaCl }\end{array}$ & $\begin{array}{l}\text { Total Cont. } \\
\text { ugNaClin }\end{array}$ \\
\hline Biokleen 5768 & 12.5 & 11.3 & 0.90 \\
1,1,1 TCA & 12.5 & 33.4 & 2.67 \\
d-limonene/IPA & 12.5 & 72.7 & 5.81
\end{tabular}




\section{Thin Film HMC's}

\begin{tabular}{|c|c|c|c|}
\hline Cleaned With & $\begin{array}{l}\text { Surface Area } \\
\text { in } \underline{2}\end{array}$ & $\begin{array}{l}\text { Total Equiv. } \\
\mu \mathrm{g} \mathrm{NaCl}\end{array}$ & $\begin{array}{l}\text { Total Cont. } \\
\mu \mathrm{g} \mathrm{NaCl} / \mathrm{n}^{2}\end{array}$ \\
\hline d-limonene & 7.5 & 13.1 & 1.75 \\
\hline d-limonene & 12.9 & 13.4 & 1.04 \\
\hline d-limonene & 7.6 & 26.8 & 3.50 \\
\hline Biokleen 5768 & 7.6 & 8.1 & 1.06 \\
\hline Biokleen 5768 & 7.6 & 10.1 & 1.32 \\
\hline Biokleen 5768 & 7.6 & 26.1 & 3.41 \\
\hline \multicolumn{3}{|c|}{ Repeat - 30 Minute Run } & \\
\hline Biokleen 5768 & 7.6 & 1.1 & 0.14 \\
\hline
\end{tabular}

